\title{
COVID-19 variant radiological findings with high lightening other coronavirus family (SARS and MERS) findings: radiological impact and findings spectrum of corona virus (COVID-19) with comparison to SARS and MERS
}

Marian Fayek Kolta* (i) and Mai Bahgat Ibrahim Ghonimy

\begin{abstract}
Background: Chest $\mathrm{CT}$ is remarkably considered as an imminent diagnostic tool and follow-up study in pulmonary changes in COVID-19 patients; being familiar to other coronavirus family CT findings, this improve our diagnostic experience and hence enhance our ability to early diagnose and combat the outbreak of COVID-19. The purpose is to investigate the wide spectrum of radiological pulmonary changes in COVID-19 patients and compare them to the variable CT findings reported in MERS and SARS.

Results: From March 15 to May 12, 2020, 50 patients in Cairo, Egypt, who have positive RT-PCR tests, were included in our study. MSCT of the chest was performed to all patients and processed in a separate work station. Two experienced radiologists assessed each study for the type and location of different pulmonary affection.

The most imminent radiological finding was patchy peripheral subpleural ground glass opacity found in 42 patients ( $84 \%$ of cases), followed by consolidation found in 30 patients (60\% of cases) and ground glass and consolidation together found in 22 patients (44\% of cases).

Unlike SARS, where initial chest imaging abnormalities are more frequently unilateral, COVID-19 is more likely to involve both lungs on initial imaging presented as bilateral peripheral subpleural scattered ground-glass opacities. Pleural effusion is absent in COVID-19 patients while it is not rare in MERS and might be observed in 20-33\% of affected individuals.

Conclusion: The imaging features of COVID-19 pneumonia are highly sensitive mainly in the outbreak pandemic. The imaging features of SARS, MERS, and COVID-19 overlap, but differences still exist especially early in disease course.
\end{abstract}

\footnotetext{
* Correspondence: Marian.fayek@hotmail.com

Radiology Department, Thoracic Imaging Unit, Faculty of medicine, Kasr Al-Aini, Cairo University, Cairo, Egypt
}

\section{Springer Open}

(c) The Author(s). 2020 Open Access This article is licensed under a Creative Commons Attribution 4.0 International License which permits use, sharing, adaptation, distribution and reproduction in any medium or format, as long as you give appropriate credit to the original author(s) and the source, provide a link to the Creative Commons licence, and indicate if changes were made. The images or other third party material in this article are included in the article's Creative Commons licence, unless indicated otherwise in a credit line to the material. If material is not included in the article's Creative Commons licence and your intended use is not permitted by statutory regulation or exceeds the permitted use, you will need to obtain permission directly from the copyright holder. To view a copy of this licence, visit http://creativecommons.org/licenses/by/4.0/. 


\section{Background}

The 2019 novel coronavirus (2019-nCoV) is a new pandemic disease diagnosed at the late 2019 in China, Wuhan. Chest CT is a key component of the diagnostic work-up for patients with suspected infection [1].

Radiological examinations are vital in early diagnosis and assessment of disease course, as most COVID-19 infected patients were diagnosed with pneumonia and characteristic CT imaging patterns [1].

In absence of specific therapeutic drugs or vaccines for 2019 novel coronavirus disease (COVID-19), it is essential to detect the diseases at an early stage and immediately isolate the infected person from the healthy population [2].

The low sensitivity of RT-PCR implies that many COVID-19 patients may not be identified and may not receive appropriate treatment in time; such patients constitute a risk for infecting a larger population given the highly contagious nature of the virus [3].

Chest $\mathrm{CT}$, as a routine imaging tool for pneumonia diagnosis, is relatively easy to perform and can produce fast diagnosis [4].

Investigators are making every effort to further characterize the imaging features of this novel coronavirus syndrome, but information is still limited [1].

Chest CT is a conventional, non-invasive imaging modality with high accuracy and speed. Based on available data published in recent literature, almost all patients with COVID-19 had characteristic CT features in the disease process [2].

The chest CT scans showed a higher sensitivity for the diagnosis of COVID-19 infection than initial RT-PCR results [2].

Similar pulmonary syndromes have been recognized as being caused by other strains of the coronavirus family. The most striking examples are the severe acute respiratory syndrome (SARS) and the Middle East respiratory syndrome (MERS) [5].

Imaging is a critical component of the diagnostic workup, monitoring of disease progression, and followup in coronavirus-related pulmonary affection [6].

Since the etiologic and clinical features of the syndrome are similar to those of SARS and MERS, the experience from those pulmonary syndromes can be helpful for managing the emerging COVID-19 outbreak [5].

The aim of this study is to familiarize radiologists with the imaging spectrum of coronavirus syndromes and to discuss the reported imaging features of COVID-19 comparing them to SARS and MERS findings.

\section{Methods}

This cross section study included 50 patients (43 males, 7 females) with age range from 32 to 75 years (mean age of 47.2 years) confirmed to be infected with SARS-CoV-
2, referred for multislice CT (MSCT) assessment of the chest (Table 1). MSCT of the chest was done to all patients as requested. The study was conducted between March 15 and May 12, 2020, in Cairo, Egypt.

\section{Inclusion criteria}

The inclusion criterion is laboratory proven PCR positive COVID-19 tests.

\section{Exclusion criteria}

The exclusion criteria are as follows:

Pregnant females

Patients presenting with acute heart failure

Patients who recently experienced clinically defined pulmonary infection attributable to other pathogens

Patients with severe artifacts on CT images

\section{Methods}

All enrolled patients were subjected to as follows:
* Through history taking.
* Laboratory assessment (CBC, ESR, and PCR).

MSCT of the chest was done to all patients using a multi-detector CT scanner with 64 or more channels. The detailed parameters for CT acquisition were as follows: tube voltage, $120 \mathrm{kVp}$; tube current, standard (reference mAs, 60-120) to low dose (reference mAs, 30) with automatic exposure control; slice thickness, 1.0 $\mathrm{mm}$; reconstruction interval, $1.0-3.0 \mathrm{~mm}$; and a sharp reconstruction kernel. CT images were obtained with the patient in the supine position at full inspiration, head first and without contrast medium (Table 2).

- Then, the images acquired sent to a separate workstation to be processed, manipulated, and reconstructed.

* Images are reconstructed in axial, coronal, and sagittal planes to detect the distribution of parenchymal affection (2D multiplanar images reconstruction, MPR). * All images were viewed on both lungs (width, 1500 $\mathrm{HU}$; level, $-700 \mathrm{HU}$ ) and mediastinal (width, $350 \mathrm{HU}$; level, $40 \mathrm{HU}$ ) settings.

Table 1 Parameters of MSCT of the chest

\begin{tabular}{ll}
\hline Tube voltage & $120 \mathrm{kVp}$ \\
\hline Tube current & $60-120 \mathrm{mAp}$ \\
Slice thickness & $1 \mathrm{~mm}$ \\
Reconstruction interval & $1 \mathrm{~mm}$ \\
Patient position & Supine \\
Respiration & Breath hold full inspiration \\
Matrix size & $512 \times 512$ \\
\hline
\end{tabular}


Table 2 Different spectrum of radiological features

\begin{tabular}{lll}
\hline Radiological feature & Number of patients & Percent of patients (\%) \\
\hline Ground glass & 42 & 84 \\
Consolidation & 30 & 60 \\
Ground glass and consolidation & 22 & 44 \\
Bilateral affection & 38 & 76 \\
Peripheral affection & 42 & 84 \\
Coarse basal pulmonary interstitium & 8 & 16 \\
Basal curvilinear atelectasis & 7 & 14 \\
Reversed halo sign & 3 & 6 \\
Crazy paving pattern & 3 & 6 \\
Mild bronchiectatic & 2 & 4
\end{tabular}

* For each patient, the chest CT scan was evaluated by two radiologists separately searching for the following characteristics: (1) presence of ground-glass opacities, (2) presence of consolidation, (3) laterality of groundglass opacities and consolidation, (4) presence of nodules, (5) presence of a pleural effusion, (6) presence of thoracic lymphadenopathy (defined as lymph node size of $\geq 10 \mathrm{~mm}$ in short-axis dimension), (7) airways abnormalities (including airway wall thickening, bronchiectasis, and endoluminal secretions), (8) axial distribution of disease (categorized as no axial distribution of disease, central "peribronchovascular" predominant disease, or peripheral predominant disease), and (9) other abnormalities, including linear opacities, opacities with a rounded morphology, opacities with a "reverse halo" sign, and opacities with a "crazy-paving" pattern.

\section{Statistical analysis}

Findings are presented as medians, and interquartile ranges due to small sample size categorical variables are described as whole numbers, with percentages in brackets.

\section{Results}

This cross section study included 50 patients (43 males, 7 females) with age ranging from 32 to 75 years (mean age of 47.2 years), with PCR positive COVID results. They were referred to perform MSCT of the chest.

Most patients presented with dyspnea, which was seen in 43 patients $(86 \%), 40$ patients suffered from fever (80\%), and 25 patients presented with dry cough (50\%).

The most imminent radiological finding was groundglass opacity found in 42 patients (84\% of cases), followed by consolidation found in 30 patients $(60 \%$ of cases) and ground glass and consolidation together found in 22 patients (44\% of cases) as shown in Table 2.

Twenty-two patients showed lower zone predominance (44\%), 18 patients showed equal distribution between the upper and lower zones (36\%), and ten patients showed upper zone predominant changes $(20 \%)$ (Table 3).

The ground-glass and consolidative opacities were peripheral in most patients with lung findings $(n=42)$, while 6 patients who had diffuse ground-glass changes and 2 patients showed peri-hilar distribution (Table 3).

Other less common findings like coarse pulmonary interstitium were in 8 patients (16\%); basal curvilinear atelectasis was seen in 7 patients (14\%); reversed halo sign was seen in three patients (6\% of cases); crazy paving pattern $(6 \%)$ and mild bronchiectatic changes were noticed in 2 patients ( $4 \%$ of cases).

Pleural effusions, pericardial effusion, cavitation, mediastinal, and hilar lymph node enlargement were not seen in any of the patients.

\section{Discussion}

Lower respiratory tract infections are the most lethal transmissible diseases worldwide, causing around 3 million deaths per year [7].

Previously, six types of coronavirus had been identified that cause human disease: four cause mild respiratory symptoms, whereas the other two, Middle East respiratory syndrome (MERS) coronavirus and severe acute respiratory syndrome (SARS) coronavirus, have caused epidemics with high mortality rates [7].

Table 3 Predominant distribution

\begin{tabular}{lll}
\hline Predominant distribution & Number of cases & Percent (\%) \\
\hline Peripheral & 42 & 84 \\
Peri-hilar & 2 & 4 \\
Diffuse & 6 & 12 \\
Upper lobar & 18 & 44 \\
Lower lobar & 22 & 36 \\
Upper and lower lobar & 10 & 20 \\
\hline
\end{tabular}


In 2019, a new strain, called SARS-CoV-2, started circulating all over the word as a pandemic, causing the disease COVID-19 [6].

Imaging is confirmed to be critical in assessing severity and disease progression in COVID-19 infection [4].

Variety of imaging features seen while studying MSCT of chest of COVID-19 patients shows great similarity to that described in other coronavirus-associated syndromes.

Sensitivity and specificity of chest CT for COVID-19 are reported to range from 80 to $90 \%$ and 60 to $70 \%$, respectively $[8,9]$.

In our study, we noted dyspnea was the commonest clinical symptoms which was in disagreement with the study done by Andrea et al. [10] who noted that fever was the most common symptoms in COVID positive patients, followed by cough and fatigue. Fever is $85.6 \%$, cough is $68.7 \%$, and fatigue is $39.4 \%$.

According to the different radiological findings in the study population, it was noted that ground-glass opacification was the most common radiological finding $84 \%$, followed by consolidation $60 \%$ which agrees with Ming et al. [11] who studied the imaging profile of the COVID-19 infection and agrees with Melina et al. [5] which showed that multifocal ground-glass opacities and consolidation were reported as main radiological features (Fig. 1).

The ground-glass and consolidative opacities were peripheral in most patients with lung findings $(n=42$, $84 \%$ ), while 6 patients had diffuse ground-glass changes and 2 patients showed peri-hilar distribution, which is matching with a study done by Ming et al. [11] who found that lung affection was peripheral in all patients with lung findings (100\%), apart from one patient who had peri-hilar ground-glass changes in addition (Figs. 2 and 3$)$.

Twenty-two patients showed lower zone predominance (44\%), 18 patients showed equal distribution between the upper and lower zones (36\%), and ten patients showed upper zone predominant changes (20\%), which is partially agreeing with Ming's study [11] who found that $44 \%$ of patients showed lower zone predominance,

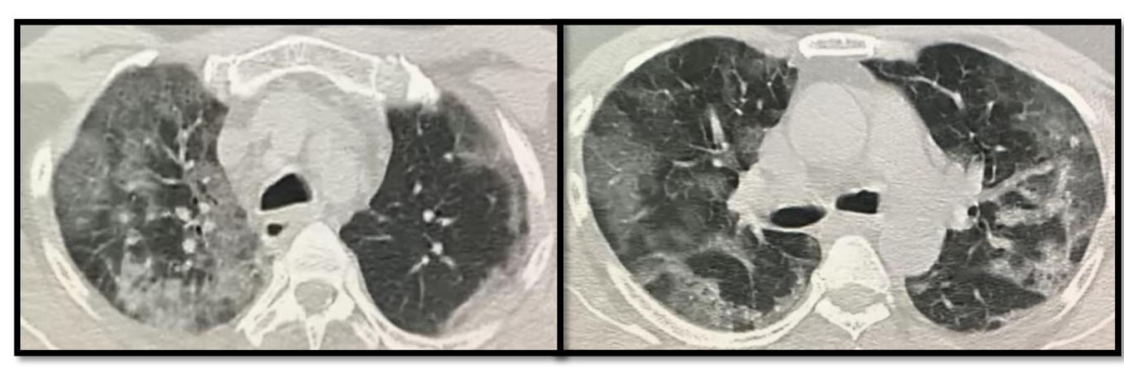

A) male patient 45 years old, presented by dyspnea and fatigue. MSCT of the chest axial cuts lung window showing: bilateral peripheral areas of ground glass attenuation and early consolidation.
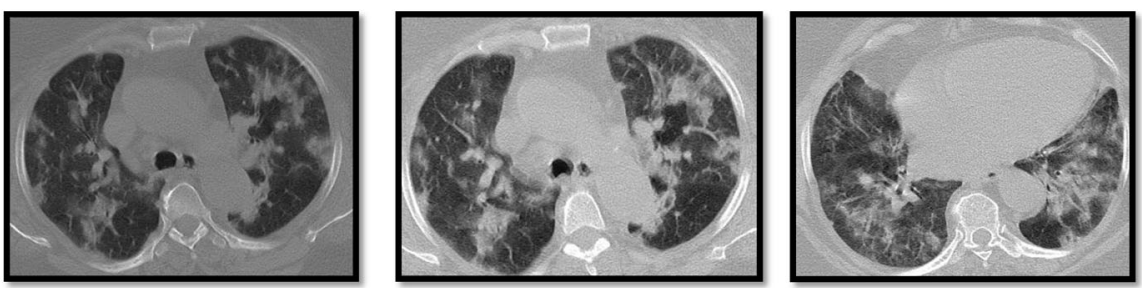

B) male patient 52 year old presented with cough, dyspnea, fever, generalized malaise and body aches. MSCT of the chest axial cuts lung window showing: bilateral scattered confluent and peripheral areas of ground glass attenuation and early consolidation

Fig. 1 a Male patient, 45 years old, presented by dyspnea and fatigue. MSCT of the chest axial cuts the lung window showing bilateral peripheral areas of ground-glass attenuation and early consolidation. b Male patient, 52 years old, presented with cough, dyspnea, fever, generalized malaise, and body aches. MSCT of the chest axial cuts the lung window showing bilateral scattered confluent and peripheral areas of ground-glass attenuation and early consolidation 


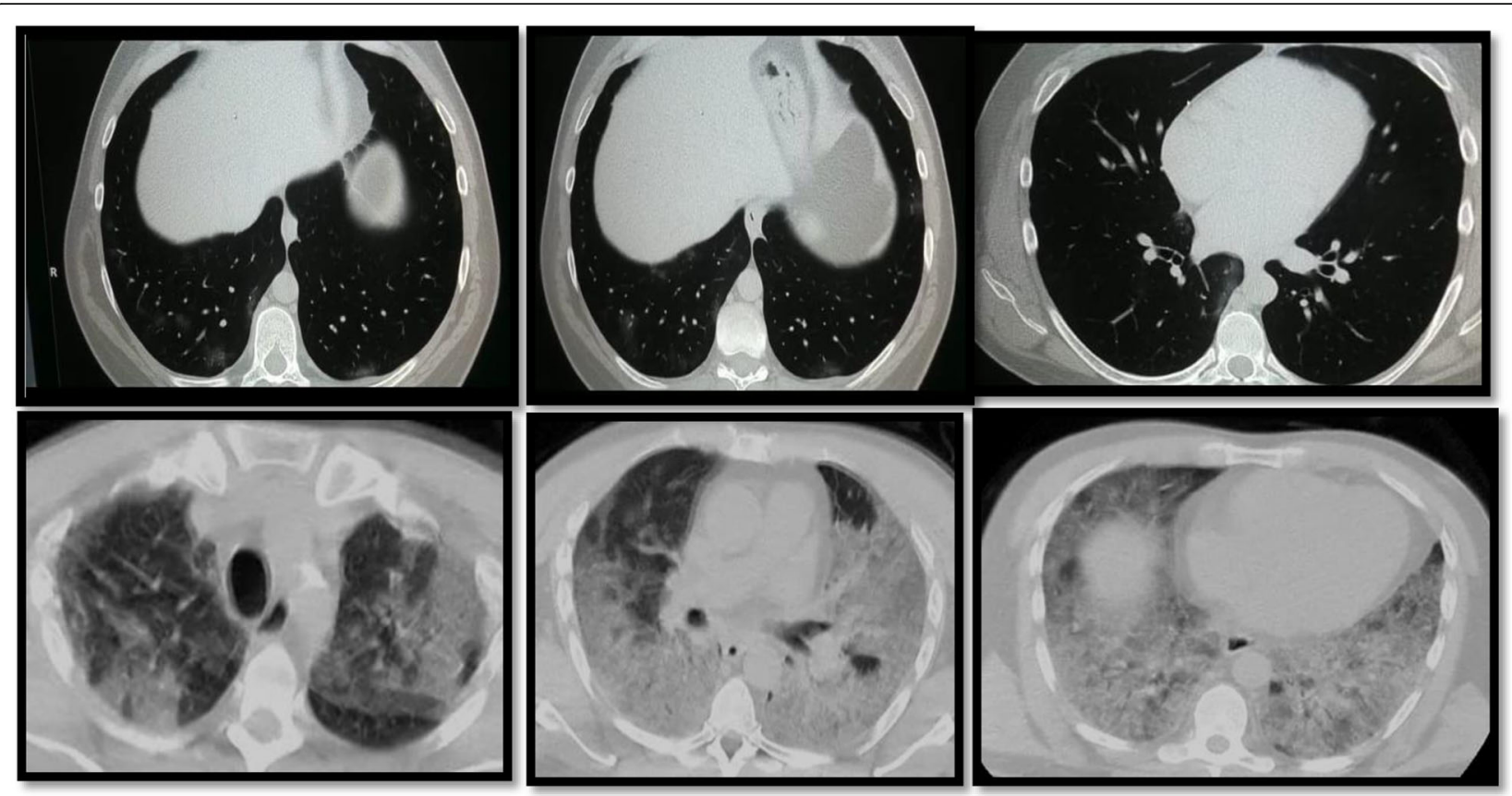

Fig. 2 A male patient, 53 years old, presented with dyspnea. MSCT chest lung window was done showing bilateral few faint mainly basal ground-glass nodular peripheral shadows. Two days later, the patient deteriorated clinically suffering severe dyspnea and fever. A follow-up MSCT chest was done showing bilateral diffuse ground glass and consolidative patches in both lung fields
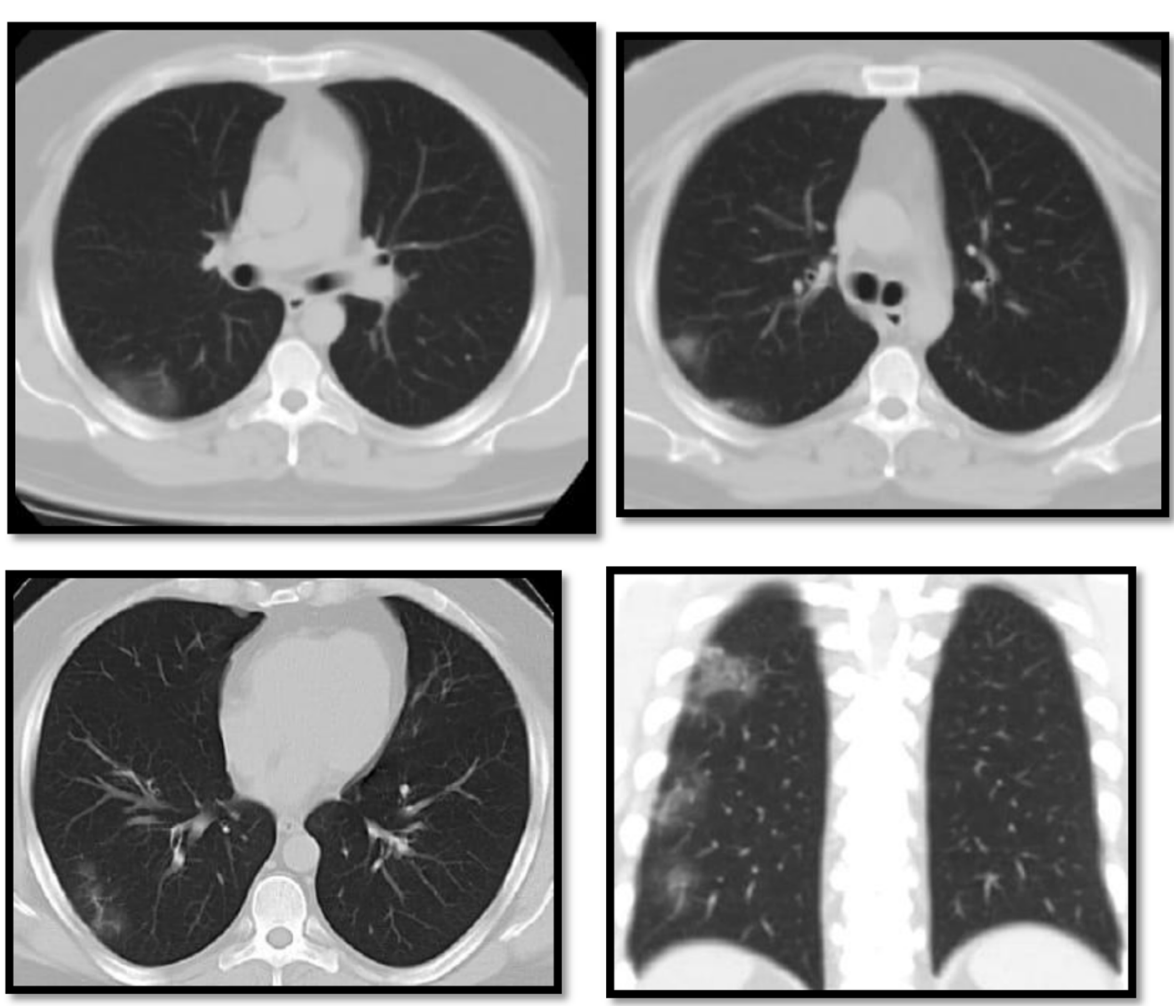

Fig. 3 A female patient, 37 years old, resented with fever and cough. MSCT of the chest was done showing typical peripheral distribution of the ground-glass opacities seen in COVID positive patients 


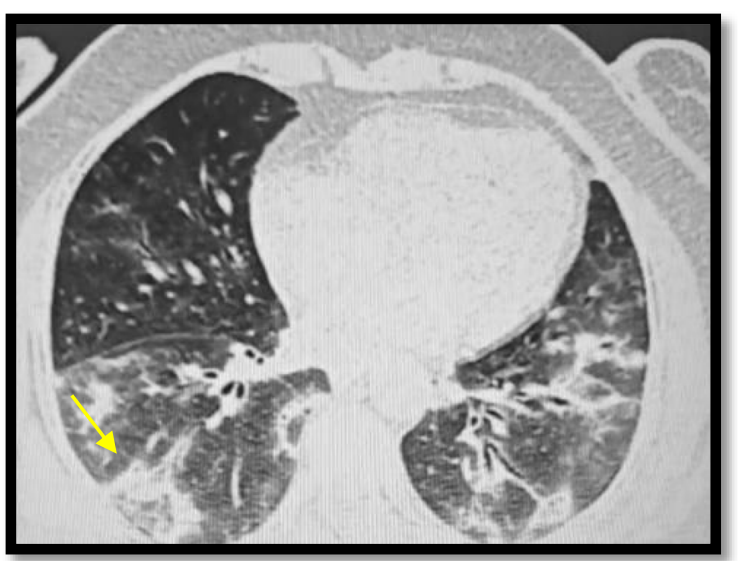

Fig. 4 A male patient, 48 years old, presented with dyspnea and fatigue. MSCT of the chest was done showing bilateral peripheral patchy areas of ground-glass attenuation, with one of them showings characteristic reversed halo sign (arrowed)

while $44 \%$ of patients showed equal distribution between the upper and lower zones and $16 \%$ of patients showed upper zone predominant changes and agrees also to the study done by Ho et al. [12], who demonstrated that the common CT findings of bilateral involvement, peripheral distribution, and lower zone dominance.
Reversed halo sign was seen in 3 cases $(6 \%)$ which is agreeing with Ming et al. who stated that reversed halo sign was seen infrequently (Fig. 4).

We found coarsening of basal pulmonary interstitium in 8 cases (16\%) which does not match with the study done by Shuchang et al. [13] who found GGO plus a reticular pattern in $62.9 \%$ of his patients (Fig. 5).

Curvilinear subpleural opacity was seen in 7 patients (14\%) which is matching with studies by $\mathrm{Wu}$ et al. [14] and $\mathrm{Li}$ et al. [15] both reported around $20 \%$ of patients with COVID-19 demonstrated this sign, which might relate to pulmonary edema or fibrosis of COVID-19 (Fig. 6).

Crazy paving pattern was seen in 3 patients $(6 \%)$ which is matching with many recent investigations reported 5 36\% COVID-19 patients with crazy paving pattern in their studies $[15,16]$. Furthermore, the presence of diffuse GGO, consolidation, and crazy paving pattern can be the signal of COVID-19 entering progressive or peak stage [17] (Fig. 7).

Pleural effusions, pericardial effusion, cavitation, mediastinal, and hilar lymph node enlargement were not seen in any of our patients which agree with Ming's study [11] which declared that lung cavitation, discrete pulmonary nodules, pleural effusions, and enlarged lymph nodes were absent.

Since COVID/SARS and MERS are considered from the same viral family (coronavirus), imaging features of

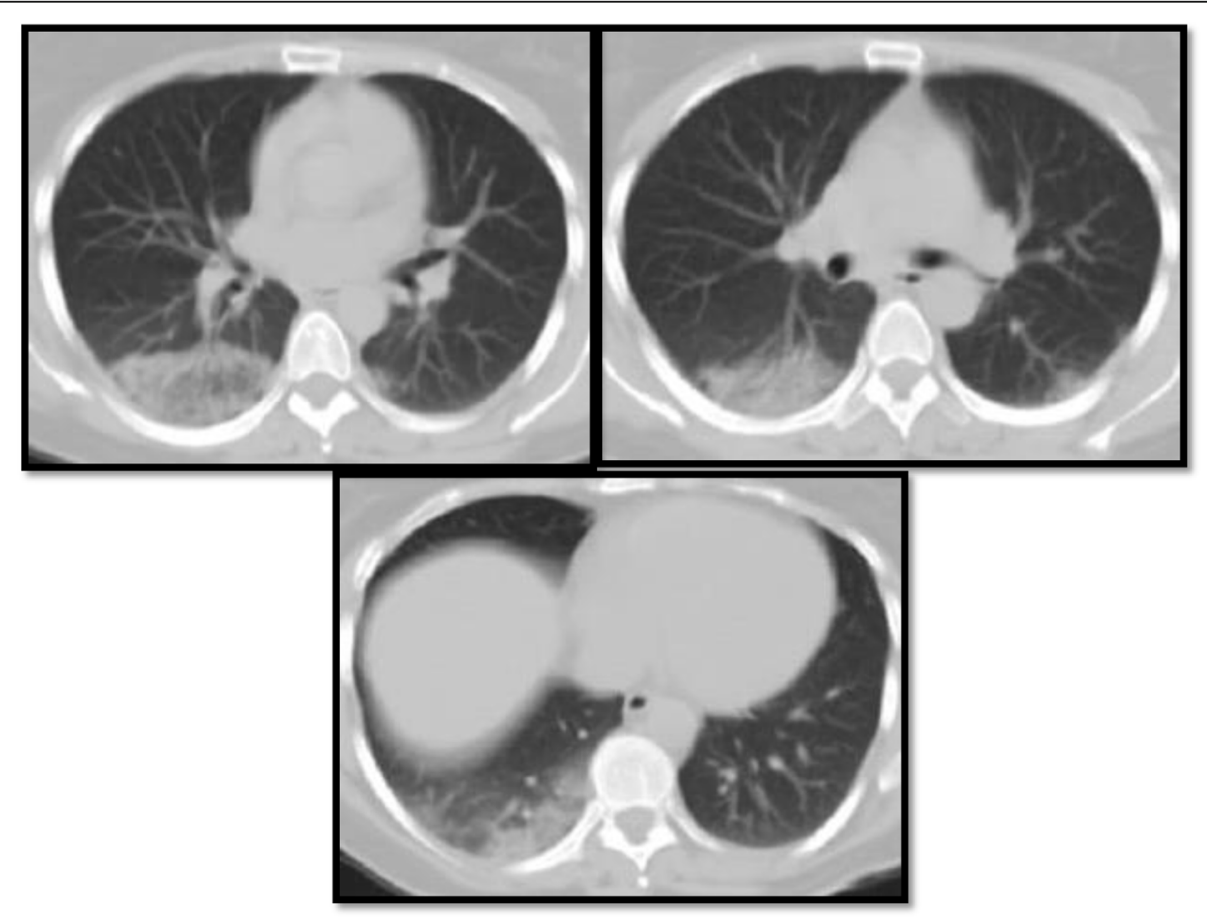

Fig. 5 A male patient, 55 years old, presented with fever and extreme fatigue. MSCT of the chest was done showing right basal coarsening of the interstitium within the ground-glass patches, which is a typical finding detected in COVID-19 viral infection 


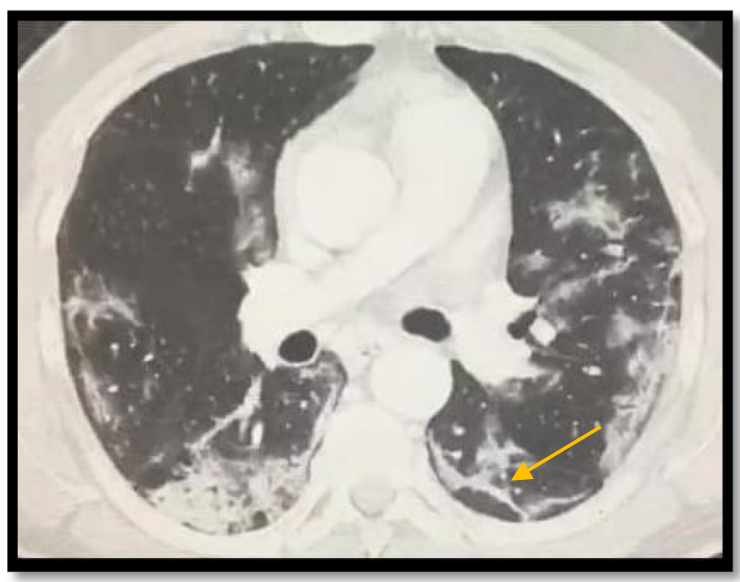

Fig. 6 Male patient, 35 years old, presented positive for COVID infection showing bilateral middle lung zones curvilinear subpleural atelectasis with peripheral consolidative patches

COVID-19, SARS, and MERS overlap, but still differences exist as well.

Unlike SARS, where initial chest imaging abnormalities are more frequently unilateral, COVID-19 is more likely to involve both lungs on initial imaging presented as bilateral peripheral subpleural scattered ground-glass opacities [5]. The majority of SARS positive patients show progressive multifocal distribution, in the followup imaging, of which $75 \%$ of patients show bilateral distribution [18], while MERS initial imaging tends to show multifocal airspace opacities in the lower lung zones which then progress to extend peri-hilar and upper lobar [19].

Pleural effusion is absent in COVID-19 patients while it is not rare in MERS and might be observed in $20-33 \%$ of affected individuals [5].

Centrilobular nodules and tree-in-bud are not characteristics of SARS or MERS [20], which is the same in COVID-19 according to our study.

Overall, the imaging findings are highly sensitive yet highly nonspecific and might overlap with the symptoms of H1N1 influenza, cytomegalovirus pneumonia, or atypical pneumonia. The acute clinical presentation and history of contact with a COVID-19-infected patient or history of recent travel should raise clinical suspicion for the diagnosis of COVID-19 [21].

\section{Conclusion}

The imaging features of COVID-19 pneumonia are highly sensitive yet nonspecific and are more often bilateral with subpleural and peripheral distribution and
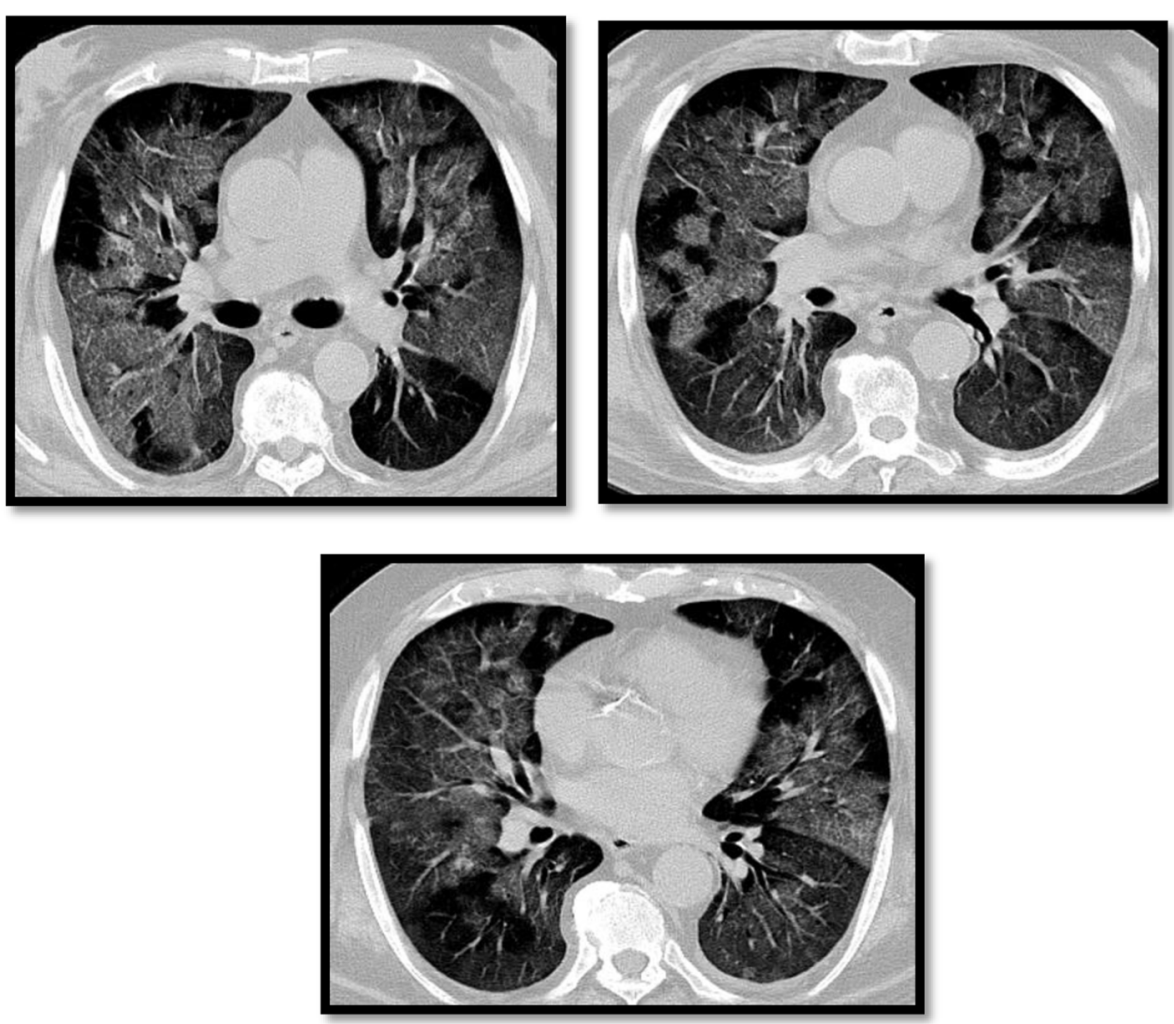

Fig. 7 A male patient, 48 years old, presented by severe respiratory distress. MSCT was done showing bilateral diffuse patchy ground-glass opacification with superimposed prominent intralobular septae giving crazy paving pattern 
range from ground-glass opacities in milder forms to consolidations in more severe forms.

The imaging features of SARS, MERS, and COVID-19 overlap, but differences exist especially early in disease course.

\section{Abbreviations}

MSCT: Multislice CT; COVID-19: Coronavirus disease 2019; RT-PCR: Real time polymerase chain reaction; SARS: Severe acute respiratory syndrome; MERS: Middle East respiratory syndrome

\section{Acknowledgements}

We all patients and their families involved in the study.

We thank all the authors for their contributions.

\section{Authors' contributions}

MG has designed the study. MF and MG contributed to the data collection. MF contributed to data analysis. MF and MG contributed to imaging data analysis and processing. All authors read and approved the final manuscript.

\section{Funding}

No source of funds.

\section{Availability of data and materials}

Data available within the article or its supplementary materials.

\section{Ethics approval and consent to participate}

We took written consent from all patients to participate in our research work.

Ethical approval was not applicable due to current situation.

\section{Consent for publication}

All authors have confirmed this article and agree to publication.

\section{Competing interests}

The authors declare that they have no conflicts of interest.

Received: 21 May 2020 Accepted: 22 July 2020

Published online: 28 August 2020

\section{References}

1. Chung M, Bernheim A, Mei X et al (2020) CT imaging features of 2019 novel coronavirus (2019-nCoV). Radiology 195:1

2. Ai T, Yang Z, Hou H et al (2020) Correlation of chest CT and RT-PCR testing in coronavirus disease 2019 (COVID-19) in China: a report of 1014 cases. Radiology 195:2

3. Huang P, Liu T, Huang L et al (2020) Use of chest CT in combination with negative RT-PCR assay for the 2019 novel coronavirus but high clinical suspicion. Radiology 10

4. Xie X, Zhong Z, Zhao W et al (2020) Chest CT for typical 2019-nCoV pneumonia: relationship to negative RT-PCR testing. Radiology 1

5. Hosseiny M, Kooraki S, Gholamrezanezhad A et al (2020) Radiology perspective of coronavirus disease 2019 (COVID-19): lessons from severe acute respiratory syndrome and Middle East respiratory syndrome. AJR 214(5):1078-1082

6. Al-Tawfiq JA, Zumla A, Memish ZA (2014) Coronaviruses: severe acute respiratory syndrome coronavirus and Middle East respiratory syndrome coronavirus in travelers. Curr Opin Infect Dis 27:411-417

7. Hayward AC, Ellen BF et al (2014) Comparative community burden and severity of seasonal and pandemic influenza: results of the Flu Watch cohort study. Lancet Respir Med 2:445-454

8. Ai T, Yang Z, Hou H, Zhan C, Chen C, Lv W, Tao Q, Sun Z, Xia L (2020) Correlation of chest CT and RT-PCR testing in coronavirus disease 2019 (COVID-19) in China: a report of 1014 cases. Radiology 200642. https://doi. org/10.1148/radiol.2020200642

9. Bai HX, Hsieh B, Xiong Z, Halsey K, Choi JW, Tran TML, Pan I, Shi LB, Wang DC, Mei J, Jiang XL, Zeng QH, Egglin TK, Hu PF, Agarwal S, Xie F, Li S, Healey T, Atalay MK, Liao WH (2020) Performance of radiologists in differentiating COVID-19 from viral pneumonia on chest CT. Radiology 200823
10. Lovato A, de Filippis C et al (2020) Clinical presentation of COVID-19: a systematic review focusing on upper airway symptoms. Ear Nose Throat $J$

11. Ng M-Y, Elaine YP et al (2020) Imaging profile of the COVID-19 infection: radiologic findings and literature review. AJR 214

12. Ho Y, Frank $W$ et al. Frequency and distribution of chest radiographic findings in COVID-1. Radiology 2020; 214.

13. Zhou S, Wang Y et al (2020) CT Features of coronavirus disease 2019 (COVID-19) pneumonia in 62 patients in Wuhan, China. AJR 8

14. Wu J, Wu X, Zeng W et al (2020) Chest CT findings in patients with corona virus disease 2019 and its relationship with clinical features. Investig Radiol

15. Kunhua Li JW, Wu F, Guo D, Chen L, Zheng F, Li C (2020) The clinical and chest CT features associated with severe and critical COVID-19 pneumonia. Investig Radiol

16. Bernheim A, Mei X, Huang M et al (2020) Chest CT findings in coronavirus disease-19 (COVID-19): relationship to duration of infection. Radiology.

17. Pan F, Ye T, Sun P et al (2020) Time course of lung changes on chest CT during recovery from 2019 novel coronavirus (COVID-19) pneumonia. Radiology.

18. Wong KT, Antonio GE, Hui DS et al (2003) Severe acute respiratory syndrome: radiographic appearances and pattern of progression in 138 Patients. Radiology 228:401-406

19. Das KM, Lee EY, Al Jawder SE et al (2015) Acute Middle East respiratory syndrome coronavirus: temporal lung changes observed on the chest radiographs of 55 patients. AJR 205

20. Ketai L, Paul NS, Wong KT (2006) Radiology of severe acute respiratory syndrome (SARS): the emerging pathologic-radiologic correlates of an emerging disease. J Thorac Imaging 21:276-283

21. Paul NS, Roberts H, Butany J et al (2004) Radiologic pattern of disease in patients with severe acute respiratory syndrome: the Toronto experience. Radio Graphics 24:553-563 [Crossref] [Medline] [Google Scholar]

\section{Publisher's Note}

Springer Nature remains neutral with regard to jurisdictional claims in published maps and institutional affiliations.

\section{Submit your manuscript to a SpringerOpen ${ }^{\circ}$ journal and benefit from:}

- Convenient online submission

- Rigorous peer review

- Open access: articles freely available online

- High visibility within the field

- Retaining the copyright to your article

Submit your next manuscript at $\boldsymbol{\nabla}$ springeropen.com 